\title{
Phase slip in a superfluid Fermi gas near a Feshbach resonance
}

\author{
Lan Yin* \\ School of Physics, Peking University, Beijing 100871, P. R. China \\ Ping Ao \\ Departments of Mechanical Engineering and Physics, \\ University of Washington, Seattle, WA 98195, USA
}

(Dated: July 3, 2018)

\begin{abstract}
In this paper, we study the properties of a phase slip in a superfluid Fermi gas near a Feshbach resonance. The phase slip can be generated by the phase imprinting method. Below the superfluid transition temperature, it appears as a dip in the density profile, and becomes more pronounced when the temperature is lowered. Therefore the phase slip can provide a direct evidence of the superfluid state. The condensation energy of the superfluid state can be extracted from the density profile of the phase slip, due to the unitary properties of the Fermi gas near the resonance. The width of the phase slip is proportional to the square root of the difference between the transition temperature and the temperature. The signature of the phase slip in the density profile becomes more robust across the BCS-BEC crossover.
\end{abstract}

*Electronic address: yinlan@pku.edu.cn 
A lot of effort has been concentrated on creating the superfluid state in a Fermi gas [1]. In recent years, the quantum degenerate regime was reached [2] and Feshbach resonance was observed in Fermi gases [3]. Asymmetric expansions of cold Fermi gases near the resonance were observed [4, 5], as a result of the strong interaction between atoms [5]. The molecular condensate was observed when the scattering length was tuned to the positive side of the resonance [6, 7]. Condensation of Fermion pairs was observed when the Fermi gas was fast tuned through the resonance [8, 9]. The measurement of the breathing-mode frequency was consistent with the predication of superfluid hydrodynamics, and the damping of the mode was weak at low temperatures [10]. Across the Feshbach resonance, a sudden change in the collective excitation spectrum was observed [11]. More recently, observation of the pairing gap in the radio-frequency excitation spectra of the Fermi gas was reported [12]. However, phase coherence of the wave-function, one of the most important properties of the superfluid state, has not been observed in these experiments so far. In this paper, we propose that phase coherence of the superfluid state can be observed in the phase-slip experiment.

Phase coherence is one of the key characteristics of macroscopic quantum phenomena such as Bose-Einstein condensation, superfluidity, and superconductivity. In these systems, the phase symmetry of the wavefunction is broken. When two systems are coupled together, the two order parameters interfere with each other. The Josephson effect appears when two superconductors with different phases are linked by a junction. In a system with two Bose-Einstein condensates, the interference pattern was observed upon release of the condensates [13]. In quasi-1D systems, the order parameter in the middle region vanishes when two sides of the system have different phases. This phenomenon is called phase slip. In quasi-1D superconductors, phase slip is the main reason for finite conductivity [14]. In Bose-Einstein condensates, phase slips have been observed in experiments [15, 16]. They are also often called solitons because they have the same dynamics as solitons.

In the superfluid state of the Fermi gas, the phase slip can be generated by the phase imprinting method. A pulsed laser can illuminate the system with different intensities on the left and right sides, and the pulse duration can be tuned so that for an atom the laser produces a phase difference of $\pi / 2$ or $-\pi / 2$ between the two sides. For a pair of atoms, the phase difference is twice as much, $\pi$ or $-\pi$, between the two sides of the system. As in superconductors, the order parameter in the superfluid state describes the pairing of atoms. When there is a discontinuity in the phase of a pair wave-function, the order parameter must 
vanish at the point of discontinuity to generate a phase slip. Phase imprinting methods have been successfully applied to create vortices [17, 18] and solitons [15, 16] in Bose-Einstein condensates. However, in the normal state of a Fermi gas, there is no phase coherence, and the phase imprinting does not produce any special effect. In the following, we study the properties of a phase slip in a superfluid Fermi gas near a Feshbach resonance. It is assumed that the phase step in the phase imprinting is applied along the axial direction of the trap and the phase slip is generated at the center of the trap.

In the superfluid state, the free energy of a Fermi gas is lower than that in the normal state. This energy difference is defined as the condensation energy. The total free energy $L$ is the sum of the condensation energy $L_{C}$ and the free energy of the normal state $L_{N}$,

$$
L=L_{C}+L_{N}
$$

According to the Ginzburg-Landau theory, the condensation energy can be expanded in terms of the order parameter $\phi$ which is proportional to the energy gap of the fermions,

$$
L_{C}=\int d^{3} r\left[-\frac{\hbar^{2}}{2 m} \phi^{*}(\mathbf{r}) \nabla^{2} \phi(\mathbf{r})+\alpha(\mathbf{r})|\phi(\mathbf{r})|^{2}+\frac{\beta(\mathbf{r})}{2}|\phi(\mathbf{r})|^{4}\right],
$$

where $\alpha$ and $\beta$ are coefficients. Below the transition temperature $T_{C}$, the sign of the coefficient $\alpha$ is negative and the superfluid state has lower free energy than the normal state. The free energy of the normal state is generally a function of temperature, particle density, trapping energy, and interaction energy which is usually proportional to the scattering length. The total free energy is a minimum in the space of the particle density and the superfluid order parameter,

$$
\begin{aligned}
& \frac{\delta L}{\delta n(\mathbf{r})}=0 \\
& \frac{\delta L}{\delta \phi^{*}(\mathbf{r})}=0
\end{aligned}
$$

where $n(\mathbf{r})$ is the fermion particle density at location $\mathbf{r}$. The derivative in equation (3) is taken under the constraint of constant total-particle number. The equilibrium configurations of the system can be determined from these saddle-point equations.

In current experiments, the temperature is well below the Fermi temperature $T_{F}$, and the system is often studied in the strong interaction regime where the magnitude of the scattering length can be much bigger than the interparticle distance. In this case, the 
interparticle distance becomes the most important length scale, and the thermodynamic properties of the Fermi gas are unitary [19]. To the leading order, the free energy density of the normal state is given by

$$
L_{N}=\int d^{3} r\left[\frac{\hbar^{2}}{2 m} c_{N} n^{\frac{5}{3}}(\mathbf{r})+V(\mathbf{r}) n(\mathbf{r})\right],
$$

where $V$ is the trapping potential and $c_{N}$ is a dimensionless constant. In the experiments 4 , 20], about 25 percent reduction of the energy by the interaction was observed, corresponding to $c_{N}=4.3$.

In the following, we study the phase-slip phenomenon in the unitary limit near the superfluid transition temperature, in the region which is likely to be accessible in experiments. The coefficients $\alpha$ and $\beta$ in the unitary limit are functions of the particle density $n$ and temperature $T$. Near the transition temperature, the coefficient $\alpha$ is approximately given by

$$
\alpha=c_{\alpha} k_{B}\left(T-T_{C}\right)
$$

where $c_{\alpha}$ is a dimensionless constant, $T$ is the temperature, and $k_{B}$ is the Boltzmann constant. In the unitary limit, the transition temperature is given by $k_{B} T_{C}=c_{T} \hbar^{2} n^{2 / 3} /(2 m)$, where $c_{T}$ is a dimensionless constant. The theoretical estimation of $T_{C}$ is about 0.5 to 0.2 times the Fermi temperature, and the corresponding constant $c_{T}$ is between 4.8 and 1.9 [21, 22, 23]. The coefficients $\beta$ in equation (2) is also a function of the fermion density, $\beta=c_{\beta} \hbar^{2} /\left[2 m n^{1 / 3}\right]$, where $c_{\beta}$ is a dimensionless constant. Currently there are no theoretical estimations of the constants $c_{\alpha}$ and $c_{\beta}$ for the strong interaction in the unitary limit. However, the two constants are available for the case of weak interactions 24],

$$
\begin{gathered}
c_{\alpha}=1.47 c_{T}^{2}, \\
c_{\beta}=2.94 c_{T}^{2} .
\end{gathered}
$$

The renormalization of these two constants by the strong interaction may not be significant, because the renormalization reduces the Fermi energy only by about 25 percent [4, 20].

In the unitary limit near the transition temperature, the saddle point equations (3) 44) are given by

$$
\begin{gathered}
-\frac{\hbar^{2}}{2 m} \nabla^{2} \phi(\mathbf{r})+\alpha(\mathbf{r}) \phi(\mathbf{r})+\beta(\mathbf{r})|\phi(\mathbf{r})|^{2} \phi(\mathbf{r})=0 \\
\frac{5 \hbar^{2}}{6 m} c_{N} n^{\frac{2}{3}}(\mathbf{r})+V(\mathbf{r})-\mu-\frac{\hbar^{2}}{3 m} c_{T} c_{\alpha} \frac{|\phi(\mathbf{r})|^{2}}{n^{\frac{1}{3}}(\mathbf{r})}=0
\end{gathered}
$$


where $\mu$ is the chemical potential. In equation (9), the term quartic in the order parameter has been dropped, because it is very small compared to other terms. In the ground state, the phase of the order parameter is same everywhere. Both the order parameter $\phi_{0}(\mathbf{r})$ and the fermion density $n_{0}(\mathbf{r})$ of the ground state can be determined from these equations. The order parameter of the ground state varies slowly. From equation (8), near the center of the trap, it is approximately given by

$$
\phi_{0}(\mathbf{r}) \approx \sqrt{-\frac{\alpha(0)}{\beta(0)}}=\sqrt{\frac{2 m c_{\alpha} k_{B}\left(T_{C}-T\right) n_{0}^{1 / 3}(0)}{c_{\beta} \hbar^{2}}},
$$

where the origin is located at the center of the trap and $\phi_{0}$ is chosen to be positive.

In a phase-slip configuration, the phase of the order parameter changes by $\pi$ from one side of the trap to the other side. With this boundary condition, the order parameter $\phi_{1}(\mathbf{r})$ and the fermion density $n_{1}(\mathbf{r})$ can also be obtained from the saddle-point equations (88) and (9). Near the transition temperature, the density of the order parameter $|\phi|^{2}$ is much less than the fermion density. From equation (9), the difference between the fermion density in the phase-slip configuration and that in ground state can be obtained,

$$
\Delta n(\mathbf{r}) \equiv n_{1}(\mathbf{r})-n_{0}(\mathbf{r}) \approx \frac{3 c_{T} c_{\alpha}}{5 c_{N}} \Delta n_{\phi}(\mathbf{r}),
$$

where $\Delta n_{\phi}$ is defined as $\Delta n_{\phi}(\mathbf{r}) \equiv\left|\phi_{1}(\mathbf{r})\right|^{2}-\left|\phi_{0}(\mathbf{r})\right|^{2}$.

In a quasi-1D trap, the system is tightly trapped in two directions, and the low-energy dynamics occurs in the other direction. Even in a trap which is not quasi-1D such as the one in Ref. [16], as long as the width of the phase slip is much less than the trap size, the dynamics of the phase slip is approximately one dimensional near the center of the trap. In this case, equation (8) is reduced to a one-dimensional form,

$$
-\frac{\hbar^{2}}{2 m} \frac{d^{2} \phi(x)}{d x^{2}}+\alpha(x) \phi(x)+\beta(x)|\phi(x)|^{2} \phi(x)=0,
$$

and the order parameter of the phase-slip state has an analytic expression,

$$
\phi_{1}(x) \approx \phi_{0}(0) \tanh (\kappa x),
$$

where $x$ is the coordinate along the axial direction, $\kappa \equiv \sqrt{-m \alpha(0)} / \hbar$, and $\phi_{1}(x)$ is chosen to be real. As plotted in Fig. (1D), in a phase-slip configuration, the order parameter vanishes in the middle. There is a phase difference of $\pi$ between the order parameter at one side and 
that at the other side. Far away from the middle, the magnitude of the order parameter approaches the value of the ground state. Note that the position of the phase slip does not change with time, because the state given by equation (13) does not carry any current.

Near the center, according to equation (111), the density change due to the phase slip is given by

$$
\Delta n(x) \approx-\frac{3 c_{T} c_{\alpha}}{5 c_{N}} \phi_{0}^{2}(0) \operatorname{sech}^{2}(\kappa x),
$$

and in terms of the density and temperature,

$$
\Delta n(x) \approx \frac{6 c_{T} c_{\alpha}^{2} m}{5 c_{N} c_{\beta} \hbar^{2}} k_{B}\left(T-T_{C}\right) n_{0}^{1 / 3}(0) \operatorname{sech}^{2}(\kappa x) .
$$

The magnitude of the density change $|\Delta n(x)|$ reaches the maximum at the center and vanishes far away. The width of the phase slip in the real space is of the order of $1 / \kappa$, where

$$
\kappa=\sqrt{c_{\alpha} m k_{B}\left(T_{C}-T\right)} / \hbar .
$$

In experiments, if $\kappa$ and the temperature can be measured, then the transition temperature $T_{C}$ can be determined. Right at the center, the density change is proportional to the ratio of the condensation-energy density $\mathcal{L}_{C}$ to the normal-state-energy density $\mathcal{L}_{N}$,

$$
\frac{\Delta n(0)}{n_{0}(0)} \approx-\frac{3 T_{C} \mathcal{L}_{C}(0)}{5\left(T-T_{C}\right) \mathcal{L}_{N}(0)},
$$

which is equal to

$$
\frac{\Delta n(0)}{n_{0}(0)}=\frac{6 c_{T} c_{\alpha}^{2} m k_{B}\left(T-T_{C}\right)}{5 c_{N} c_{\beta} \hbar^{2} n_{0}^{2 / 3}(0)},
$$

where $\mathcal{L}_{C}(0)=\alpha(0)\left|\phi_{0}(0)\right|^{2} / 2$ and $\mathcal{L}_{N}(0)=c_{N} n_{0}^{5 / 3}(0)$. Thus the density profile of a phase slip can provide important information about the properties of the superfluid ground state such as the transition temperature and the condensation energy.

In experiments, the method to detect the phase slip is the measurement of the density profile. However, the trap also causes the inhomogeneity of the density distribution. Both the phase slip and the trap lead to the quadratic dependence of the density on the distance away from the center, but they have different signs. Whether or not the phase slip shows up as a dip in the density profile is determined by the second derivative of the particle density at the center. The particle density can be written as the sum of the particle density of the ground-state and the density difference between the phase-slip state and the ground state,

$$
n_{1}(x)=\Delta n(x)+n_{0}(x) .
$$


The second derivative of the density change due to the phase slip is given by

$$
\Delta n^{\prime \prime}(0)=\frac{6 c_{T} c_{\alpha}}{5 c_{N}} \phi_{0}^{2}(0) \kappa^{2}=\frac{12 c_{T} c_{\alpha}^{3} m^{2}}{5 c_{N} c_{\beta} \hbar^{4}} k_{B}^{2}\left(T_{C}-T\right)^{2} n_{0}^{1 / 3}(0) .
$$

Near $T_{C}$, the density profile of the ground state is approximately given by the Thomas-Fermi approximation,

$$
\frac{5 \hbar^{2}}{6 m} c_{N} n_{0}^{\frac{2}{3}}(x)+V(x)-\mu=0,
$$

and the second derivative of the density is approximately given by

$$
n_{0}^{\prime \prime}(0)=-\frac{9 m}{5 \hbar^{2} c_{N}} n_{0}^{\frac{1}{3}}(0) V^{\prime \prime}(0)=-\frac{9 m^{2} \omega_{x}^{2}}{5 \hbar^{2} c_{N}} n_{0}^{\frac{1}{3}}(0)
$$

where $\omega_{x}$ is the axial frequency of the trap. Whether the phase slip appears as a dip in the density profile depends on the ratio of the two second derivatives,

$$
\left|\frac{\Delta n^{\prime \prime}(0)}{n_{0}^{\prime \prime}(0)}\right|=\frac{4 c_{T} c_{\alpha}^{3} k_{B}^{2}\left(T-T_{C}\right)^{2}}{3 c_{\beta} \hbar^{2} \omega_{x}^{2}} .
$$

If this ratio is bigger than one, then there is a dip in the density profile.

In the experiment by Chin et al. [12], the excitation gap was observed at the Fermi energy $E_{F} / k_{B}=3.2 \mu \mathrm{K}$ with the axial frequency $\omega_{x} /(2 \pi)=24 \mathrm{~Hz}$. The transition temperature $T_{C}$ was estimated to be about a quarter of the Fermi temperature [25], corresponding to $c_{T}=2.4$. Currently there are no better estimation of the constants $c_{\alpha}$ and $c_{\beta}$ than the values given by equations (6) and (7). With these numbers, even if the temperature is lower than the transition temperature by just one percent, $T_{C}-T=0.01 T_{C}$, the ratio of the two density derivatives given in equation (23) is already much larger than one, $\Delta n^{\prime \prime}(0) / n_{0}^{\prime \prime}(0) \approx 6.8 \times 10^{4}$. Thus the phase slip can be identified by the dip at the center of the trap in the density profile for the vast range of the temperature below the transition temperature. In Fig. (2), the density profile of a phase slip at temperature $T=0.9 T_{C}$ is plotted near the center of trap. At the center, the particle density of the phase slip is smaller than that of the ground state by about 12 percent, which is very likely to be detected in the experiment. Although better estimations of the constants such as $c_{\alpha}$ and $c_{\beta}$ can improve these calculations, but it will probably not lead to any change greater than an order of a magnitude, as the interaction only changes the normal-state energy by about 25 percent in the unitary limit [4, 20]. The measurement of the constants $c_{\alpha}$ and $c_{\beta}$ in the phase-slip experiment can also serve as a test for microscopic theories. 
The strong interaction is the reason for the unitary properties of a Fermi gas. However, when the Fermi gas is very close to a Feshbach resonance, there is a crossover from the superfluid state (BCS-type) to the molecular-condensate state (BEC). In the crossover region, fermion atoms coexist with diatomic molecules, and properties of this mixture cannot be described by the unitary properties of the fermions alone, because of the interaction between atoms and molecules. Although in the crossover region many properties of the system are unclear, the phase slip can still be generated by the phase-imprinting method, because in both the superfluid and molecular-condensate states the phase symmetry of the wavefunction is broken.

At the other side of the crossover, in the molecular-condensate state with a weak interaction, the dynamics is well described by the Gross-Pitaevski equation (GP-equation)

$$
i \hbar \frac{\partial \psi}{\partial t}=\left(-\frac{\hbar^{2}}{4 m} \nabla^{2}+V\right) \psi+g_{m}|\psi|^{2} \psi
$$

where $\psi$ is the wavefunction of the molecular condensate, $g_{m}$ is the coupling constant $g_{m}=$ $\hbar^{2} a_{m} / 2 m$, and $a_{m}$ is the scattering length of the molecules. The soliton solution of the GPequation has been well studied. Near the center of the trap, the stationary soliton solution is approximately given by

$$
\psi(x, t) \approx \psi_{0} \tanh \left(\kappa_{m} x\right) e^{-2 i \mu t / \hbar}
$$

where the chemical potential is given by $\mu=g_{m}\left|\psi_{0}\right|^{2} / 2$ and $\kappa_{m}=2 \sqrt{m \mu} / \hbar$. In this configuration, the molecular density vanishes at the center and the phase difference of the wave-function across the center is given by $\pi$. The width of the phase slip is of the order of $1 / \kappa_{m}$ from which both the coupling constant $g_{m}$ and the scattering length $a_{m}$ can be extracted. In Ref. [26], $a_{m}$ was estimated to be about 0.6 times the scattering length of the atoms. The phase slip in the molecular-condensate state is much easier to be identified in the density profile because the density vanishes at the center. Comparing the properties of the phase slip at both sides of the BEC-BCS crossover, we expect that the particle density at the center drops dramatically as the system is tuned from the superfluid state to the molecular-condensate state, due to the increase of the molecular density.

In conclusion, we predict that the phase slip can be generated in a superfluid Fermi gas by the phase imprinting technique. The presence of the phase slip can be detected by the measurement of the density profile. The phase slip can provide an excellent direct evidence 
of the superfluid state, because phase coherence is one of the key signatures of superfluidity. The density profile of a phase slip also offers important information about the superfluid state, such as the condensation energy and the transition temperature. We would like to acknowledge support from NSFC under Grant No. 90303008 and support from SRF for ROCS, SEM.

[1] L. Pitaevskii and S. Stringari, Science 298, 2144 (2002).

[2] B. DeMarco and D. S. Jin, Science 285, 1703 (1999).

[3] T. Loftus, C. A. Regal, C. Ticknor, J. L. Bohn, and D. S. Jin, Phys. Rev. Lett. 88, 173201 (2002).

[4] K. M. O’Hara, S. L. Hammer, M. E. Gehm, S. R. Granade, and J. E. Thomas, Science 298, 2179 (2002).

[5] C. A. Regal and D. S. Jin, Phys. Rev. Lett. 90, 230404 (2003).

[6] S. Jochim et al., Science 302, 2101 (2003).

[7] M. Greiner, C. A. Regal, and D. S. Jin, Nature 426, 537 (2003).

[8] C. A. Regal, M. Greiner, and D. S. Jin, Phys. Rev. Lett. 92, 040403 (2004).

[9] M. W. Zwierlein, C. A. Stan, C. H. Schunck, S. M. F. Raupach, A. J. Kerman, and W. Ketterle, Phys. Rev. Lett. 92, 120403 (2004).

[10] J. Kinast, S. L. Hemmer, M. E. Gehm, A. Turlapov, and J. E. Thomas, Phys. Rev. Lett. 92, $150402(2004)$.

[11] M. Bartenstein, A. Altmeyer, S. Riedl, S. Jochim, C. Chin, J. H. Denschlag, and R. Grimm, Phys. Rev. Lett. 92, 203201 (2004).

[12] C. Chin et al., Science 305, 1128 (2004).

[13] M. R. Andrews et al., Science 275, 637 (1997).

[14] C. N. Lau, N. Markovic, M. Bockrath, A. Bezryadin, and M. Tinkham, Phys. Rev. Lett. 87, $217003(2001)$.

[15] S. Burger et al., Phys. Rev. Lett. 83, 5198 (1999).

[16] J. Denschlag et al., Science 287, 97 (2000).

[17] J. E. Williams and M. J. Holland, Nature 401, 568 (1999).

[18] M. R. Matthews, B. P. Anderson, P. C. Haljan, D. S. Hall, C. E. Wieman, and E. A. Cornell, 
Phys. Rev. Lett. 83, 2498 (1999).

[19] T.-L. Ho, Phys. Rev. Lett. 92, 090402 (2004).

[20] M. E. Gehm, S. L. Hemmer, S. R. Granade, K. M. O'Hara, and J. E. Thomas, Phys. Rev. A 68, 011401(R) (2003).

[21] M. Holland, S. J. J. M. F. Kokkelmans, M. L. Chiofalo, and R. Walser, Phys. Rev. Lett. 87, 120406 (2001).

[22] Y. Ohashi and A. Griffin, Phys. Rev. Lett. 89, 130402 (2002).

[23] E. Timmermans et al., Phys. Lett. A 285, 228 (2001).

[24] J. R. Schrieffer, Theory of superconductivity (Benjamin, Reading, 1964), chapter 8; V. N. Popov, Functional integrals and collective excitations (Cambridge University, Cambridge, 1987), chapter 13.

[25] J. Kinnunen, M. Rodriguez, and P. Törmä, Science 305, 1131 (2004).

[26] D.S. Petrov, C. Salomon, and G.V. Shlyapnikov, Phys. Rev. Lett. 93, 090404 (2004). 


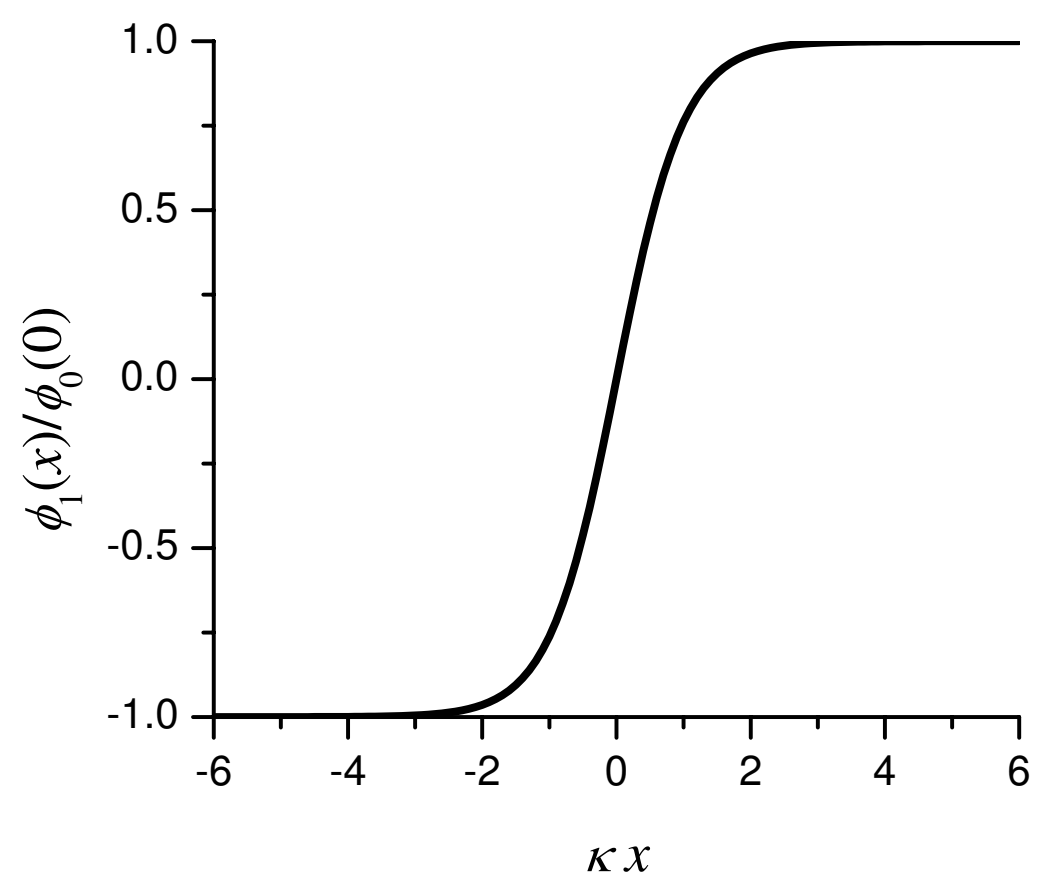

FIG. 1: The order parameter $\phi_{1}(x)$ of a phase-slip configuration near the center of trap. The width of the phase slip is much smaller than the trap size. The order parameter vanishes in the middle. Across the middle, the phase difference of the order parameter is given by $\pi$. Away from the center, the magnitude of the order parameter approaches the value of the ground state. 


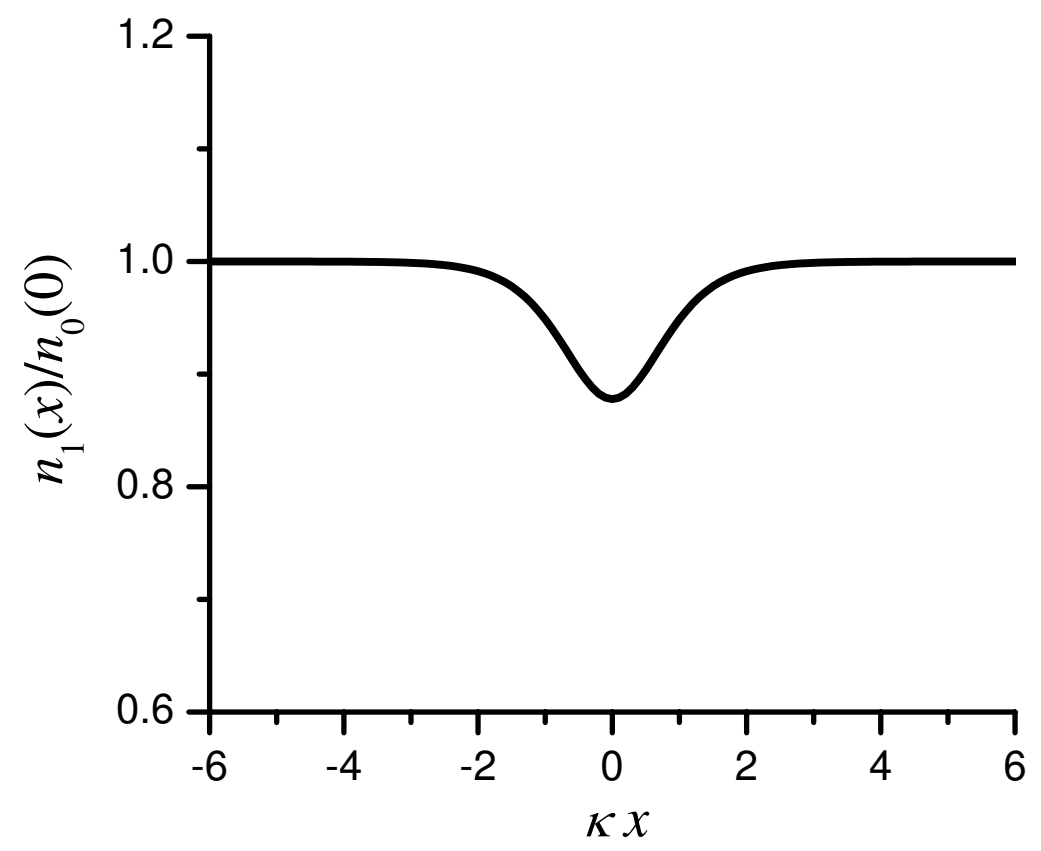

FIG. 2: The density profile of a phase slip for the experimental conditions given in Ref. [12] at the temperature $T=0.9 T_{C}$, where $T_{C} \approx 0.25 T_{F}, T_{F}=3.4 \mu \mathrm{K}$, and $\omega_{x}=24 \mathrm{~Hz}$. The units of the two axes are given by $1 / \kappa \approx 1 /\left[0.39 n_{0}^{\frac{1}{3}}(0)\right]$ and $n_{0}(0) \approx 3.3 \times 10^{13} / \mathrm{cm}^{3}$. The value of $n_{0}(0)$ is calculated from the Thomas-Fermi approximation. The width of the phase slip is of the order of $1 / \kappa$ which is much smaller than the trap size. 